\title{
Cortical plasticity after brachial plexus injury and repair: a resting-state functional MRI study
}

\author{
Dhananjaya I. Bhat, MCh, ${ }^{1}$ B. Indira Devi, MCh, ${ }^{1}$ Komal Bharti, MTech, ${ }^{1}$ and Rajanikant Panda, ME² \\ Departments of ${ }^{1}$ Neurosurgery and ${ }^{2}$ Neuroimaging and Interventional Radiology, National Institute of Mental Health and \\ Neurosciences (NIMHANS), Bangalore, India
}

\begin{abstract}
OBJECTIVE The authors aimed to understand the alterations of brain resting-state networks (RSNs) in patients with pan-brachial plexus injury (BPI) before and after surgery, which might provide insight into cortical plasticity after peripheral nerve injury and regeneration.

METHODS Thirty-five patients with left pan-BPI before surgery, 30 patients after surgery, and 25 healthy controls underwent resting-state functional MRI (rs-fMRI). The 30 postoperative patients were subdivided into 2 groups: 14 patients with improvement in muscle power and 16 patients with no improvement in muscle power after surgery. RSNs were extracted using independent component analysis to evaluate connectivity at a significance level of $p<0.05$ (familywise error corrected).
\end{abstract}

RESULTS The patients with BPI had lower connectivity in their sensorimotor network (SMN) and salience network (SN) and greater connectivity in their default mode network (DMN) before surgery than the controls. Connectivity of the left supplementary motor cortex in the SMN and medial frontal gyrus and in the anterior cingulate cortex in the SN increased in patients whose muscle power had improved after surgery, whereas no significant changes were noted in the unimproved patients. There was a trend toward reduction in DMN connectivity in all the patients after surgery compared with that in the preoperative patients; however, this result was not statistically significant.

CONCLUSIONS The results of this study highlight the fact that peripheral nerve injury, its management, and successful treatment cause dynamic changes within the brain's RSNs, which includes not only the obvious SMN but also the higher cognitive networks such as the SN and DMN, which indicates brain plasticity and compensatory mechanisms at work.

https://thejns.org/doi/abs/10.3171/2016.12.FOCUS16430

KEY WORDS brachial plexus injury; cortical plasticity; default mode network; fMRI; functional connectivity; independent component analysis; peripheral nerve injury; resting state; salience network; sensorimotor network

$\mathrm{B}$ RACHIAL plexus injury (BPI) is a peripheral nerve injury (PNI) that can result in a severe and disabling complete loss of motor and sensory function of the affected upper limb and can be associated with deafferentation pain. Such injuries are associated with significant morbidity and psychological stress, all of which result in reduced quality of life. ${ }^{24}$ Despite improvements in our understanding of the pathophysiology of nerve injury, repair, and regeneration and improvements in microsurgical techniques, the results of surgery to treat this injury have been far from satisfactory. ${ }^{16}$ Gaining insight into the changes in the brain that take place after these events might help us to develop new strategies for improving outcome after management of such injuries. Using task-based functional MRI (fMRI), transcranial magnetic stimulation, and intraoperative electrophysiological studies, cortical plasticity (or cortical functional reorganization) has been seen in the sensorimotor cortex after PNI and its recovery in humans and in animal experiments. . $^{17,20,29,36}$

It is now known that after PNI, there is a loss of afferent signals to the sensorimotor cortex and a rapid change in the somatotopic organization of body parts in both the sensory and motor cortices occur. The functions of the surrounding normal cortices encroach on the defunct cortex,

ABBREVIATIONS BPI = brachial plexus injury; DMN = default mode network; FDR = false discovery rate; fMRI = functional MRI; FWE = familywise error; ICA = independent component analysis; MNI 152 = Montreal Neurological Institute 152; MRC = Medical Research Council; PNI = peripheral nerve injury; rsFC = resting-state functional connectivity; rs-fMRI = resting-state fMRI; RSN = resting-state network; SMN = sensorimotor network; SN = salience network.

SUBMITTED October 20, 2016. ACCEPTED December 21, 2016.

INCLUDE WHEN CITING DOI: 10.3171/2016.12.FOCUS16430. 
which results in expansion of the surrounding somatotopic map into the defunct zone. After recovery, there is another reorganization, but the new map will not be the same as the original map because of peripheral mismatch in the regeneration of nerve fibers. ${ }^{5,6,9,13-15,18,19,30}$

Resting-state fMRI (rs-fMRI) is a novel method of examining the functional networks of the brain and is beneficial because it does not depend on task performances, it allows data to be easily acquired, and it enables the evaluation of functional networks. ${ }^{2,732,38}$ Although task-based fMRI cannot be performed for patients with BPI because their affected limb is completely paralyzed, rs-fMRI analysis can be done without patients performing any task. Thus, rs-fMRI shows tremendous potential for increasing our understanding of cortical functions in nerve injury and repair. Decreased interhemispheric functional connectivity between motor cortices in patients with complete unilateral BPI before surgery, revealed through the use of seed-based rs-fMRI, has been found in a few studies. ${ }^{17,28}$ Qiu et al. ${ }^{29}$ used a seed-based rs-fMRI approach to demonstrate reduced resting-state functional connectivity (rsFC) between the supplementary motor area and motor cortical areas associated with the hand and arm contralateral to the injured side. This improvement over time despite no improvement in motor power was attributed to expansion of the adjacent cortical areas that encroach into the cortical areas that control the hand and arm. The results of these studies indicate that rs-fMRI is useful for assessing the dynamic changes that take place in the brain after PNI.

In this study, we aimed to understand dynamic alterations in the resting-state networks (RSNs) that might occur after BPI and repair. We hypothesized that after BPI, there is a loss of motor and sensory functions that results in disturbed rsFC involving the sensorimotor cortex, and either direct or indirect changes in the cognitive association networks along with the SMN can occur and might be attributed to psychological stress. Our second aim was to use rs-fMRI to gain an understanding of cortical plasticity and brain reorganization after surgery in patients with BPI.

\section{Methods}

\section{Patient Selection}

The study group consisted of consecutive patients who were previously in good health and had sustained a panBPI or complete BPI to the left upper limb for which surgery was performed between 2012 and 2015 at the National Institute of Mental Health and Neurosciences in India. Patients with head and/or and spine injury were excluded from the study. The demographic data, clinical findings, surgical procedure, and postoperative improvement during follow-up were recorded. The surgical procedure performed was intercostal musculocutaneous nerve anastomosis with sural nerve graft, which was followed by regular physiotherapy. ${ }^{23,36}$ The surgery was performed after the induction of general anesthesia. The musculocutaneous nerve was exposed in the arm proximal to its entry into the biceps, followed by the exposure of any two adjacent intercostal nerves under the third to fifth ribs. The intercostal nerves were cut, and the proximal end was anastomosed to the musculocutaneous nerve using the su- ral nerve harvested from the ipsilateral leg. Preoperative and postoperative rs-fMRI was performed in all patients. Handedness-, age-, and sex-matched healthy individuals were used as controls.

This study was approved by the National Institute of Mental Health and Neurosciences Human Ethical Committee. Written consent was obtained from each study subject.

\section{Data Acquisition}

Structural and rs-fMRI were performed in each subject. The rs-fMRI data were acquired using a 3-T Skyra MRI (Siemens) scanner with a 32-channel coil. A total of 185 volumes of spin-echo echo-planar images were obtained using the following parameters: TR $3000 \mathrm{msec}$; TE 35 msec; 36 slices with 4-mm thickness; field of view $192 \times 192 \mathrm{~mm}$; matrix $64 \times 64$; and voxel size $3 \times 3 \times 3$ $\mathrm{mm}$. After rs-fMRI, T1-weighted magnetization-prepared rapid gradient-echo (MP-RAGE) data were acquired with 192 slices in a single volume with a TR of $1600 \mathrm{msec}$, a TE of $0.35 \mathrm{sec}$, and a voxel size of $1 \times 1 \times 1 \mathrm{~mm}$.

\section{Data Analysis}

The rs-fMRI data were preprocessed using FSL software (https://fsl.fmrib.ox.ac.uk/fsl/fslwiki). The preprocessing steps included nonbrain tissue extraction, ${ }^{33}$ motion correction, slice-timing correction, and 5-mm full width at half-maximum spatial smoothing. The functional images were coregistered with the structural image in Montreal Neurological Institute 152 (MNI 152) standard space, and the data were resampled to 2-mm resolution., ${ }^{2,732-34,38}$ Followed by the preprocessing steps, independent component analysis (ICA) was performed to extract RSNs and exclude the noise components for subjects by multivariate exploratory linear optimized decomposition into independent components (MELODIC). ${ }^{4,34}$ The noise components were examined visually and excluded using the fsl_regfilt function in FSL.

After individual subject analysis, multivariate group probability ICA was performed using MELODIC to derive maximally and spatially independent components across all subjects. The rs-fMRI data were decomposed into 15 sets of independent component vectors., ${ }^{44}$ Dualregression analysis was performed for group-wise comparisons of independent components, which enabled voxel-wise comparisons. ${ }^{3,11}$ Statistical differences were assessed using randomized nonparametric permutation, and the threshold-free cluster-enhancement technique was incorporated by performing 5000 random permutations. ${ }^{26,35}$ To estimate group mean effects, the results were threshold with familywise error (FWE) corrected at $\mathrm{p} \leq 0.05$, and intergroup effects were threshold with false-discovery rate (FDR) corrected at $\mathrm{p} \leq 0.05 .^{11}$

\section{Results}

Thirty-five patients with left BPI and a mean $( \pm$ SD) age of $22 \pm 4.32$ years were included in the study. A total of 42 patients underwent surgery to treat a left BPI during the study period, but 7 patients refused to participate in the study. Every patient was male. The most common mode 
of injury was an accident while riding on a motor bike (31 patients), 3 patients fell from a height, and 1 patient sustained injury as a result of falling from a moving train. All the patients were right handed and had sustained a panBPI; they each had limp upper-left limb strength rated as a Medical Research Council (MRC) grade of 0/5 in all muscles groups in that limb. Results of electroneuromyography in all these patients were suggestive of preganglionic injury; hence, none of them underwent exploration of the brachial plexus, but peripheral intercostal musculocutaneous nerve coaptation was performed for each of them. We advise shoulder joint arthrodesis if a patient has persistent shoulder dislocation; however, none of these patients required such an intervention. The mean $( \pm S D)$ interval between injury and surgery was $6.05 \pm 1.34$ months. We followed up with the patients every 3 months, and clinical recovery was seen in the sixth and ninth months of follow-up for most patients. Each patient underwent postoperative rs-fMRI once. Five follow-up fMRI studies were discarded because of the long spans (20-24 months) between surgery and postoperative MRI or artifacts in data. The remaining 30 postoperative patients were divided into 1 of 2 groups: 1) those who experienced improvement in muscle power after surgery (elbow flexion MRC grade of at least $3 / 5$ ) or 2) those whose muscle power had not improved after surgery (elbow flexion MRC grade of $0 / 5-2 / 5)$. A total of 14 patients showed improvement after surgery (mean age $21.85 \pm 4.7$ years; mean time between injury and surgery $6.5 \pm 1.4$ months), and 16 patients did not show improvement after surgery (mean age $20.75 \pm$
3.69 years; mean time between injury and surgery $5.87 \pm$ 1.35 months). MRI was performed $7 \pm 1.8$ months after surgery (see Supplemental Table 1). Twenty-five healthy male age-matched right-handed volunteers with no previous history of brain insult, PNI, or psychiatric illness were recruited (mean age 22.61 \pm 5.13 years) as controls

From 15 independent components, we found differences in 3 that corresponded to the sensorimotor network (SMN), the default mode network (DMN), and the salience network (SN). We analyzed the group mean effects of controls, preoperative patients with BPI, postoperative patients with improvement, and postoperative patients without improvement.

\section{Sensorimotor Network}

Less rsFC in the SMN was noted in the patients with BPI than in the controls. The bilateral precentral gyrus and postcentral gyrus of the SMN network showed less rsFC in patients with BPI before surgery than that in controls. In the postoperative groups, SMN rsFC increased significantly $(\mathrm{p}<0.05)$ in the left postcentral gyrus in postoperative patients with improvement in muscle power, whereas no change in SMN rsFC was seen in the postoperative patients who experienced no improvement in muscle power (Fig. 1; Table 1).

\section{Salience Network}

The SN showed less rsFC in patients with BPI before surgery than in the controls. After surgery, we observed a
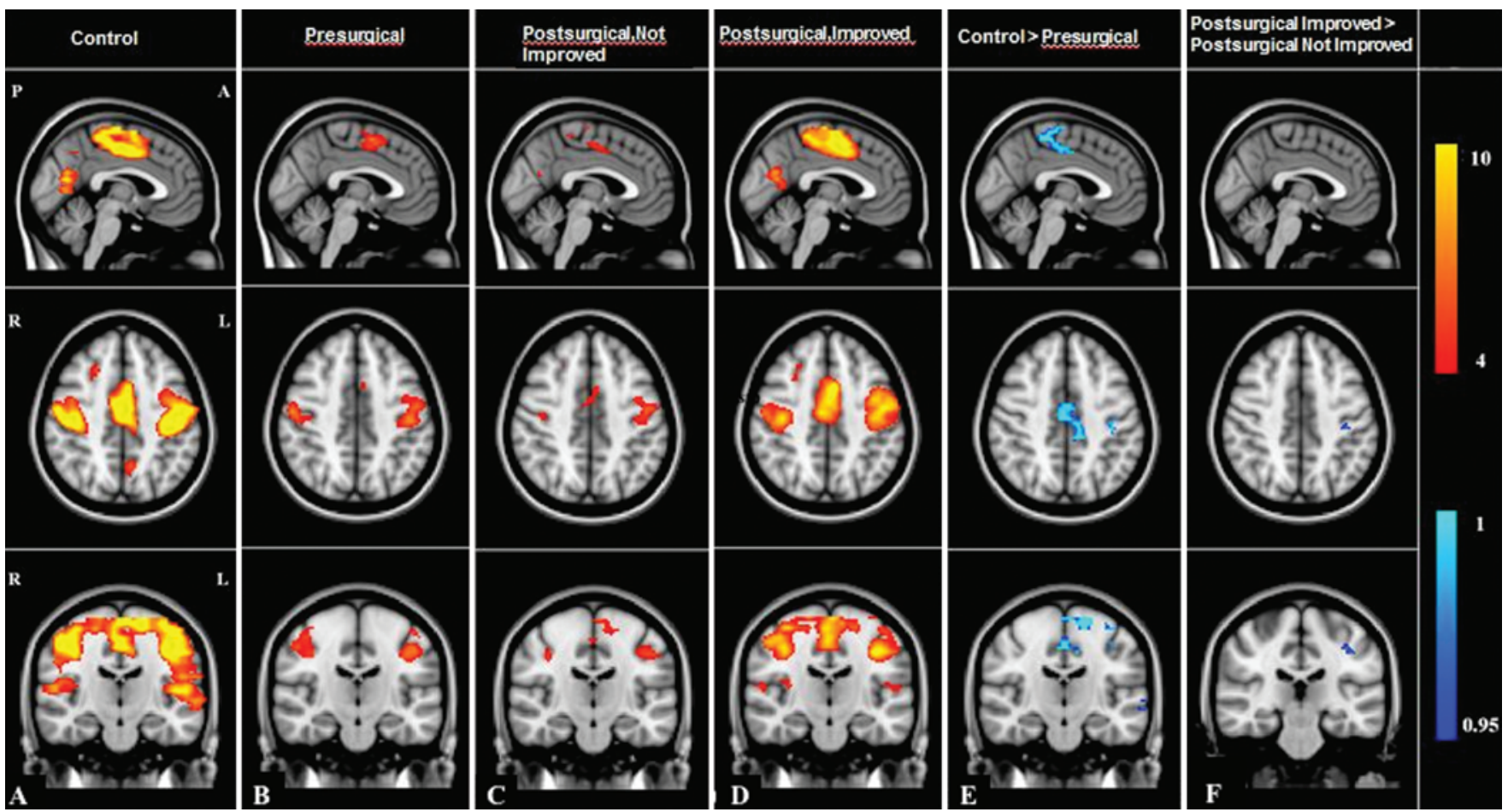

FIG. 1. Functional MR images of the SMN in healthy controls (A), preoperative patients (B), postoperative patients who showed no improvement (C), postoperative patients who did show improvement (D), and healthy controls versus preoperative patient with $\mathrm{BPI}$ (indicating that patients with BPI have decreased connectivity in the SMN) (E) and subtracted functional MR images of postoperative patients who showed no improvement versus postoperative patients who did show improvement (indicating increased connectivity in the SMN in postoperative patients who showed improvement) (F). $A=$ anterior; $L=$ left; $P=$ posterior; $R=$ right. 
TABLE 1. Group mean effects on SMN images in the 4 study groups

\begin{tabular}{|c|c|c|c|c|c|c|}
\hline \multirow[b]{2}{*}{ Group } & \multirow[b]{2}{*}{ Brain Region } & \multicolumn{3}{|c|}{ Coordinate } & \multirow{2}{*}{$\begin{array}{c}\text { Cluster } \\
\text { Voxel }\end{array}$} & \multirow{2}{*}{$\begin{array}{l}\text { Peak } \\
\text { z-Stat* }\end{array}$} \\
\hline & & $x$ & $y$ & z & & \\
\hline \multirow[t]{6}{*}{ Control } & Postcentral gyrus, It & -42 & -22 & 40 & 2,673 & 10.4 \\
\hline & Postcentral gyrus, rt & 40 & -22 & 44 & 1,634 & 9.7 \\
\hline & Precentral gyrus, It & -30 & -26 & 62 & 1,986 & 8.3 \\
\hline & Precentral gyrus, rt & 21 & -30 & 62 & 1,396 & 8.2 \\
\hline & Supplementary motor area, It & -2 & 3 & 51 & 783 & 8 \\
\hline & Supplementary motor area, rt & 3 & 4 & 52 & 782 & 8.1 \\
\hline \multirow[t]{5}{*}{ Presurgical } & Postcentral gyrus, It & -42 & -22 & 40 & 466 & 7.6 \\
\hline & Postcentral gyrus, rt & 58 & -2 & 32 & 559 & 7.3 \\
\hline & Precentral gyrus, It & -42 & -6 & 43 & 102 & 6.8 \\
\hline & Supplementary motor area, It & -2 & 9 & 52 & 282 & 6.1 \\
\hline & Supplementary motor area, rt & 6 & 6 & 60 & 224 & 6.3 \\
\hline \multirow[t]{5}{*}{ Postsurgical, not improved } & Postcentral gyrus, It & -50 & -10 & 36 & 548 & 6.7 \\
\hline & Postcentral gyrus, rt & 59 & -6 & 28 & 330 & 5.8 \\
\hline & Precentral gyrus, It & -55 & -4 & 28 & 385 & 5.1 \\
\hline & Precentral gyrus, rt & 58 & 3 & 28 & 101 & 4.6 \\
\hline & Supplementary motor area, It & -13 & -12 & 61 & 34 & 4.1 \\
\hline \multirow[t]{6}{*}{ Postsurgical, improved } & Postcentral gyrus, It & 40 & -20 & 43 & 1,540 & 8.1 \\
\hline & Postcentral gyrus, rt & 41 & -21 & 43 & 933 & 7.9 \\
\hline & Precentral gyrus, It & -18 & -24 & 61 & 1,652 & 7.8 \\
\hline & Precentral gyrus, rt & 26 & -24 & 60 & 1,196 & 7.9 \\
\hline & Supplementary motor area, It & -6 & -9 & 60 & 648 & 8.5 \\
\hline & Supplementary motor area, rt & 2 & -10 & 52 & 606 & 9.6 \\
\hline \multirow[t]{4}{*}{ Control > presurgical } & Postcentral gyrus, It & -36 & -26 & 49 & 102 & 0.983 \\
\hline & Postcentral gyrus, rt & 28 & -26 & 58 & 69 & 0.982 \\
\hline & Precentral gyrus, It & -23 & -27 & -60 & 89 & 0.993 \\
\hline & Precentral gyrus, rt & 14 & -30 & 64 & 56 & 0.992 \\
\hline Postsurgical, improved > postsurgical, not improved & Postcentral gyrus, It & -39 & -26 & 42 & 39 & 0.992 \\
\hline
\end{tabular}

significant increase in rsFC in clinically improved postsurgical patients over that of presurgical patients with BPI and the controls. We found no significant changes in the postsurgical patients without improvement. The anterior cingulate gyrus, right superior frontal gyrus, and left insular cortex showed weaker rsFC in patients with BPI than in the controls. The bilateral middle frontal gyrus and right superior frontal gyrus showed greater rsFC in the postsurgical patients with improvement than in the postsurgical patients without improvement and the preoperative patients (Fig. 2; Table 2).

\section{Default Mode Network}

We found greater rsFC in the DMN in patients with BPI than in the controls. They had a higher rsFC in the posterior cingulate cortex and precuneus. The mean group image maps of DMN showed a trend toward decreased rsFC after surgery, but we found no significant changes in the DMN between the preoperative and postoperative patient groups (Fig. 3; Table 3).

\section{Discussion}

To our knowledge, this study is the first to have evaluated changes in RSNs in patients with BPI before and after surgical repair. Overall, we observed alterations in rsFC in the SMN, DMN, and SN before and after surgery and compared rsFC with that in healthy controls. We tried to identify any differences in these networks between patients who had improved muscle power after surgery and those who did not. We found changes in the SMN of patients with BPI before and after surgery and differences between muscle power in the improved and unimproved groups, as well. These results can be attributed to cortical plasticity; however, what is interesting is that although there is no primary insult to the brain after PNI, changes in higher cognitive networks (DMN and $\mathrm{SN}$ ) were found in these patients with BPI. Not only were these changes seen before surgery, but dynamic changes were also observed after surgery, especially in the group of patients whose muscle power had improved.

SMN is a well-studied RSN, and its major subareas in- 

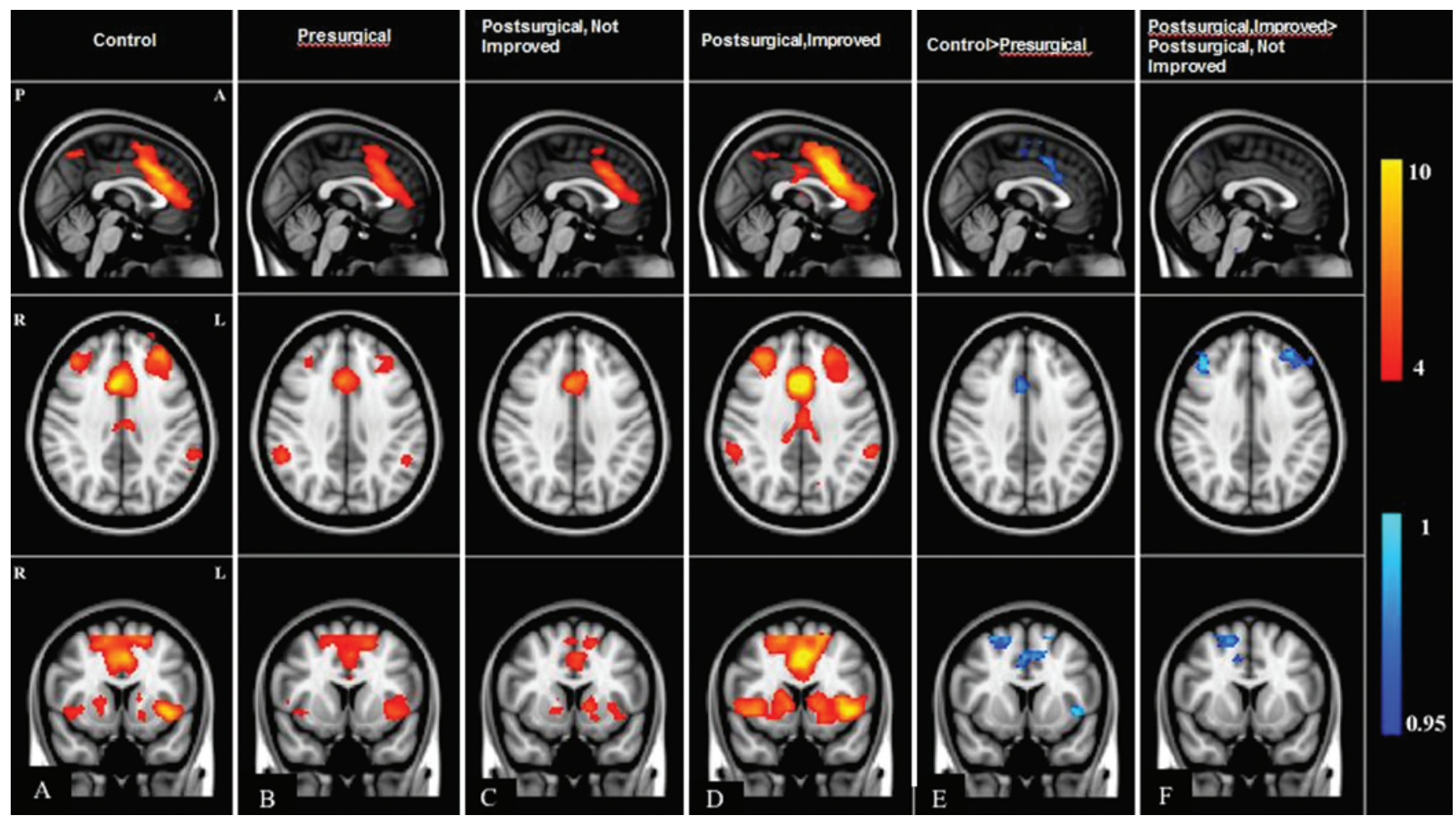

FIG. 2. Functional $M R$ images of the $S N$ in a healthy control individual $(A)$, a preoperative patient $(B)$, a postoperative patient who showed no improvement (C), a postoperative patient who did show improvement (D), and a healthy control versus a preoperative patient with BPI (indicating that patients with BPI have decreased connectivity in the SN) (E) and subtracted functional MR images of a postoperative patient who showed no improvement versus a postoperative patient who did show improvement (indicating increased connectivity in the SN in postoperative patients who showed improvement) (F).
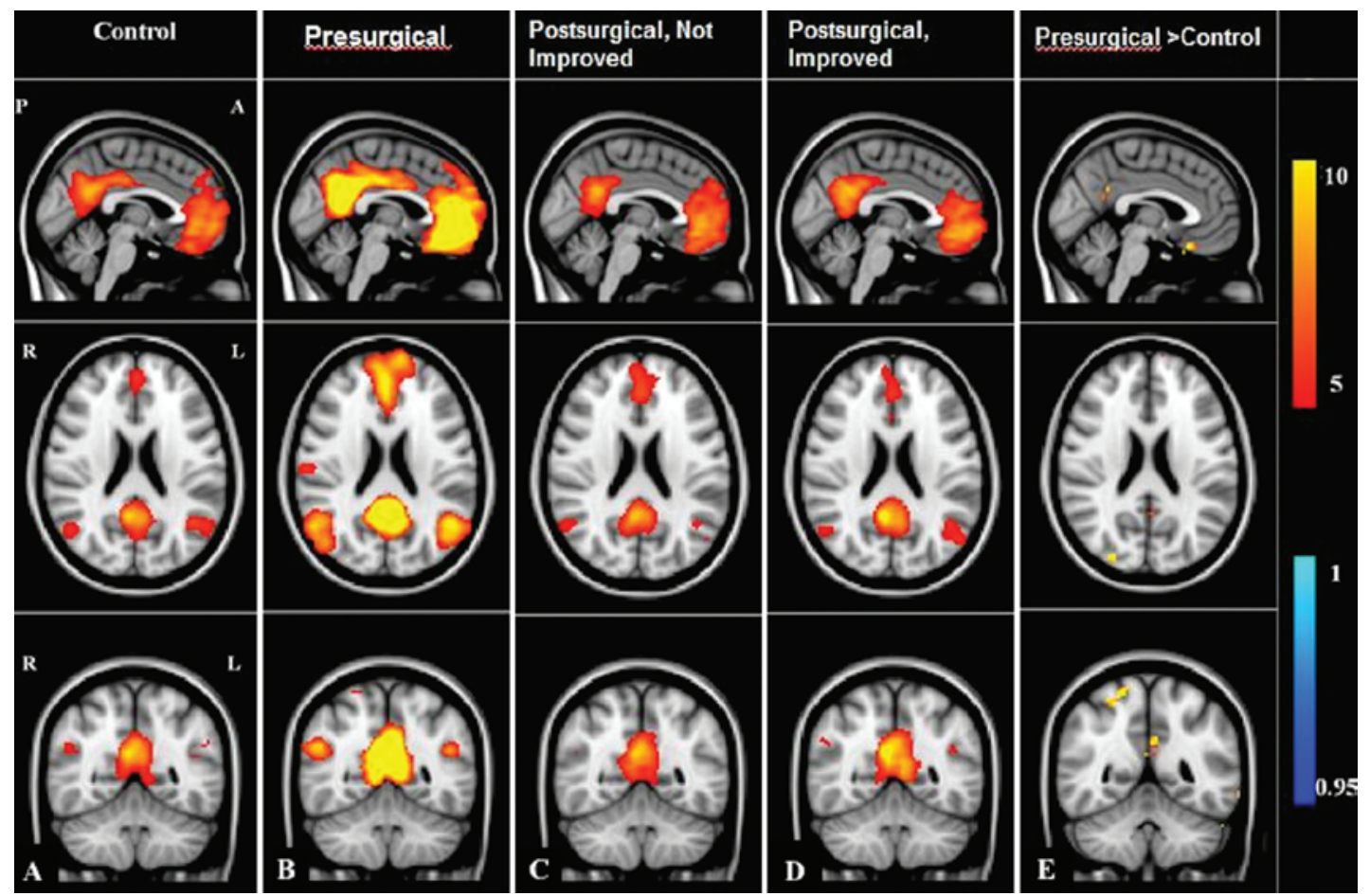

FIG. 3. Functional MR images of the DMN in a healthy control individual $(\mathbf{A})$, a preoperative patient $(\mathbf{B})$, a postoperative patient who showed no improvement (C), and a postoperative patient who did show improvement (D) and subtracted functional MR images of a healthy control individual versus a preoperative patient with BPI (indicating that patients with BPI have increased connectivity in the DMN) (E). 
TABLE 2. Group mean effects on SN images in the 4 study groups

\begin{tabular}{|c|c|c|c|c|c|c|}
\hline \multirow[b]{2}{*}{ Group } & \multirow[b]{2}{*}{ Brain Region } & \multicolumn{3}{|c|}{ Coordinate } & \multirow{2}{*}{$\begin{array}{l}\text { Cluster } \\
\text { Voxel }\end{array}$} & \multirow{2}{*}{$\begin{array}{l}\text { Peak } \\
\text { z-Stat }\end{array}$} \\
\hline & & $x$ & $y$ & z & & \\
\hline \multirow[t]{12}{*}{ Control } & Insula, It & -38 & 14 & -5 & 578 & 11.2 \\
\hline & Insula, rt & 33 & 18 & -4 & 333 & 7.2 \\
\hline & Putamen, It & -17 & 8 & -5 & 189 & 11.2 \\
\hline & Putamen, rt & 24 & 10 & -4 & 172 & 7.2 \\
\hline & Cingulum, anterior, rt & 10 & 23 & 23 & 1,925 & 10.7 \\
\hline & Cingulum, middle, It & 5 & 14 & 36 & 1,036 & 9.9 \\
\hline & Frontal, middle, It & -47 & 44 & 16 & 1,688 & 10.1 \\
\hline & Frontal, middle, rt & 38 & 44 & 16 & 1,329 & 9.7 \\
\hline & Frontal, superior, It & -23 & 44 & 16 & 888 & 9.1 \\
\hline & Frontal, superior, rt & 30 & 51 & 13 & 554 & 9.8 \\
\hline & Supramarginal, It & -58 & -42 & 29 & 202 & 6.3 \\
\hline & Supramarginal, rt & 55 & -42 & 28 & 202 & 5.8 \\
\hline \multirow{12}{*}{$\begin{array}{l}\text { Presurgi- } \\
\text { cal }\end{array}$} & Insula, It & -37 & 14 & -5 & 527 & 8.8 \\
\hline & Insula, rt & 43 & 15 & -6 & 274 & 6.5 \\
\hline & Putamen, It & -18 & 17 & -6 & 47 & 8.7 \\
\hline & Putamen, rt & 29 & 15 & 3 & 21 & 6.2 \\
\hline & Cingulum, anterior, rt & 5 & 34 & 14 & 1,378 & 8.8 \\
\hline & Cingulum, middle, It & 8 & 18 & 36 & 660 & 7.9 \\
\hline & Frontal, middle, It & -32 & 42 & 22 & 1,611 & 8.4 \\
\hline & Frontal, middle, rt & 30 & 34 & 28 & 985 & 7.3 \\
\hline & Frontal, superior, It & -20 & 49 & 22 & 525 & 8.7 \\
\hline & Frontal, superior, rt & 18 & 55 & 18 & 269 & 7.1 \\
\hline & Supramarginal, It & -50 & -46 & 37 & 84 & 5.2 \\
\hline & Supramarginal, rt & 55 & -42 & 32 & 311 & 7.3 \\
\hline \multirow{12}{*}{$\begin{array}{l}\text { Postsurgi- } \\
\text { cal, not } \\
\text { im- } \\
\text { proved }\end{array}$} & Insula, It & -42 & 18 & -5 & 411 & 6.8 \\
\hline & Insula, rt & 41 & 14 & -4 & 215 & 7.2 \\
\hline & Putamen, It & -18 & 11 & -2 & 223 & 6.1 \\
\hline & Putamen, rt & 19 & 11 & -2 & 109 & 7 \\
\hline & Cingulum, anterior, rt & 6 & 32 & 19 & 1,204 & 7.6 \\
\hline & Cingulum, middle, It & 8 & 18 & 36 & 611 & 6.9 \\
\hline & Frontal, middle, It & -31 & 43 & 24 & 1,158 & 7.8 \\
\hline & Frontal, middle, rt & 26 & 42 & 28 & 714 & 5.9 \\
\hline & Frontal, superior, It & -21 & 55 & 16 & 329 & 7.6 \\
\hline & Frontal, superior, rt & 27 & 55 & 18 & 202 & 6.3 \\
\hline & Supramarginal, It & -58 & -42 & 28 & 66 & 4.8 \\
\hline & Supramarginal, rt & 54 & -42 & 28 & 119 & 5.5 \\
\hline \multirow{12}{*}{$\begin{array}{l}\text { Postsurgi- } \\
\text { cal, im- } \\
\text { proved }\end{array}$} & Insula, It & -38 & 10 & -4 & 750 & 12.3 \\
\hline & Insula, rt & 38 & 10 & -4 & 540 & 9.7 \\
\hline & Putamen, It & -17 & 8 & -5 & 468 & 12 \\
\hline & Putamen, rt & 20 & 10 & -4 & 357 & 9.1 \\
\hline & Cingulum, anterior, rt & 10 & 35 & 13 & 2,451 & 13.1 \\
\hline & Cingulum, middle, It & 11 & 18 & 36 & 1,811 & 12.4 \\
\hline & Frontal, middle, It & -42 & 45 & 19 & 1,973 & 11.3 \\
\hline & Frontal, middle, rt & 38 & 45 & 18 & 1,747 & 11.8 \\
\hline & Frontal, superior, It & -23 & 58 & 18 & 1,196 & 13.1 \\
\hline & Frontal, superior, rt & 31 & 59 & 12 & 1,029 & 12.7 \\
\hline & Supramarginal, It & -58 & -38 & 32 & 266 & 6 \\
\hline & Supramarginal, rt & 54 & -42 & 28 & 385 & 7.2 \\
\hline
\end{tabular}

CONTINUED IN NEXT COLUMN »
» CONTINUED FROM PREVIOUS COLUMN

TABLE 2. Group mean effects on SN images in the 4 study groups

\begin{tabular}{|c|c|c|c|c|c|c|}
\hline \multirow[b]{2}{*}{ Group } & \multirow[b]{2}{*}{ Brain Region } & \multicolumn{3}{|c|}{ Coordinate } & \multirow{2}{*}{$\begin{array}{l}\text { Cluster } \\
\text { Voxel }\end{array}$} & \multirow{2}{*}{$\begin{array}{l}\text { Peak } \\
\text { z-Stat }\end{array}$} \\
\hline & & $\mathrm{x}$ & $y$ & $\mathrm{z}$ & & \\
\hline \multirow{3}{*}{$\begin{array}{c}\text { Control > } \\
\text { presur- } \\
\text { gical }\end{array}$} & $\begin{array}{l}\text { Anterior cingulate } \\
\text { gyrus }\end{array}$ & 11 & 23 & 25 & 104 & 9.83 \\
\hline & $\begin{array}{l}\text { Superior frontal } \\
\text { gyrus, rt }\end{array}$ & 33 & 50 & 14 & 78 & 0.981 \\
\hline & Insular cortex, It & -34 & 12 & -5 & 47 & 0.981 \\
\hline \multirow{3}{*}{$\begin{array}{c}\text { Postsurgi- } \\
\text { cal, im- } \\
\text { proved } \\
\text { > post- } \\
\text { surgical, } \\
\text { not im- } \\
\text { proved }\end{array}$} & $\begin{array}{l}\text { Middle frontal gyrus, } \\
\text { It }\end{array}$ & -40 & 42 & 22 & 164 & 9.81 \\
\hline & $\begin{array}{l}\text { Middle frontal gyrus, } \\
\mathrm{rt}\end{array}$ & 35 & 47 & 16 & 113 & 0.982 \\
\hline & $\begin{array}{l}\text { Superior frontal } \\
\text { gyrus, rt }\end{array}$ & 30 & 56 & 15 & 52 & 0.981 \\
\hline
\end{tabular}

Group mean effects derived from SN images in the 4 groups (control; presurgical; postsurgical, not improved; and postsurgical, improved) were FWE corrected at $p \leq 0.05$ for multiple comparisons; between-group contrasts (control $>$ presurgical; postsurgical, improved > postsurgical, not improved) were FDR corrected at $p<0.05$. Coordinates were extracted from MNI 152 space.

clude the precentral gyrus, postcentral gyrus, and supplementary motor area. Using seed-based methods to identify the SMN, Liu et al. ${ }^{17}$ and Min et al..$^{22}$ found an absence of motor area activity on the side contralateral to the limb affected by BPI. Liu et al. ${ }^{17}$ found decreased interhemispheric rsFC between motor cortices in patients with BPI. No studies have documented postoperative changes in this network. We observed rsFC in the bilateral precentral gyrus, in both the posterior and anterior cingulate gyrus, and postcentral gyrus in the SMN in patients that was lower than that in controls, which indicates decreased rsFC attributable to deafferentation. SMN rsFC reformation in the group of postoperative patients who showed improvement was much higher than that in the postoperative patients who showed no improvement and in the preoperative patients but less than that in the controls. Why there was increased connectivity in the left supplementary motor cortex (ipsilateral to the side of injury) is difficult to explain and probably reflects the overactivity of the dominant lobe after injury and recovery.

The $\mathrm{SN}$ is a principal functional network that plays an important role in attention, cognitively relevant events, and the subsequent engagement of frontoparietal systems for working memory and higher-order cognitive control. Anatomically, the SN includes the dorsal anterior cingulate cortex, frontoinsular cortex, and limbic structures. ${ }^{21}$ The SN has been studied extensively in patients with neuropsychiatric illness, which affects cognition, and a considerable decrease in the rsFC of this network has been demonstated. ${ }^{1}$ No studies have evaluated alterations in the SN after PNI. Ros et al. ${ }^{31}$ noted an increase in SN connectivity after neurofeedback training. They found neurobehavioral evidence for the brain's exquisite functional plasticity and for a temporally direct impact of neurofeedback on a key cognitive control network. In our study, we found decreased $\mathrm{rsFC}$ in the anterior cingulate gyrus of the $\mathrm{SN}$ in patients 
TABLE 3. Group mean effects on DMN images in the 4 study groups

\begin{tabular}{|c|c|c|c|c|c|c|}
\hline \multirow[b]{2}{*}{ Group } & \multirow[b]{2}{*}{ Brain Region } & \multicolumn{3}{|c|}{ Coordinate } & \multirow{2}{*}{$\begin{array}{l}\text { Cluster } \\
\text { Voxel }\end{array}$} & \multirow[b]{2}{*}{ T-Max } \\
\hline & & $x$ & $y$ & $z$ & & \\
\hline \multirow{6}{*}{ Control } & Posterior cingulate cortex & 6 & -54 & 24 & 1,579 & 7.6 \\
\hline & Medial frontal gyrus & -2 & 38 & -12 & 4,400 & 7.3 \\
\hline & Angular gyrus, It & -42 & -70 & 36 & 673 & 9 \\
\hline & Angular gyrus, rt & 54 & -62 & 32 & 533 & 7.5 \\
\hline & Middle temporal gyrus, It & -62 & -10 & -24 & 409 & 7.6 \\
\hline & Middle temporal gyrus, rt & 58 & -2 & -28 & 216 & 6.3 \\
\hline \multirow{6}{*}{ Presurgical } & Posterior cingulate cortex & 6 & -54 & 20 & 2,428 & 12.8 \\
\hline & Medial frontal gyrus & -6 & 46 & 4 & 5,163 & 9.7 \\
\hline & Angular gyrus, It & -46 & -62 & 28 & 605 & 7.6 \\
\hline & Angular gyrus, rt & 54 & -58 & 28 & 420 & 8 \\
\hline & Middle temporal gyrus, It & -58 & -22 & -20 & 318 & 7.9 \\
\hline & Middle temporal gyrus, rt & 62 & -6 & -24 & 65 & 5.1 \\
\hline \multirow{6}{*}{$\begin{array}{l}\text { Postsurgical, not } \\
\text { improved }\end{array}$} & Posterior cingulate cortex & 6 & -54 & 24 & 1,539 & 7.8 \\
\hline & Medial frontal gyrus & -2 & 38 & -12 & 3,846 & 7.3 \\
\hline & Angular gyrus, It & -50 & -66 & 32 & 486 & 7 \\
\hline & Angular gyrus, rt & 54 & -78 & 28 & 228 & 5.2 \\
\hline & Middle temporal gyrus, It & -58 & -6 & -24 & 151 & 4.1 \\
\hline & Middle temporal gyrus, rt & 58 & -10 & -28 & 145 & 5.4 \\
\hline \multirow{6}{*}{$\begin{array}{l}\text { Postsurgical, im- } \\
\text { proved }\end{array}$} & Posterior cingulate cortex & 6 & -54 & 24 & 2,028 & 7.9 \\
\hline & Medial frontal gyrus & -2 & 38 & -12 & 4,109 & 7.6 \\
\hline & Angular gyrus, It & -42 & -70 & 36 & 605 & 7.3 \\
\hline & Angular gyrus, rt & 54 & -62 & 32 & 420 & 7.9 \\
\hline & Middle temporal gyrus, It & -62 & -10 & -24 & 318 & 7.8 \\
\hline & Middle temporal gyrus, rt & 58 & -2 & -28 & 65 & 5.0 \\
\hline \multirow{2}{*}{ Presurgical > control } & Posterior cingulate cortex & 6 & -54 & 24 & 43 & 0.983 \\
\hline & Medial frontal gyrus & -2 & 38 & -12 & 12 & 0.981 \\
\hline
\end{tabular}

with BPI before surgery. The increased rsFC in the bilateral middle frontal gyrus and right superior frontal gyrus in patients who improved after surgery points toward brain plasticity and an inherent effort of the brain to form networks that restore functionality. Further longitudinal study might help us to gain a deeper understanding of the SN in therapeutics and interventions and to determine if this gain in functionality has a graded response.

The DMN is another network that has been studied extensively with rs-fMRI and has been shown to be reliably present in most neuroscience studies. Its subarea includes the posterior cingulate cortex, ventromedial prefrontal cortex, and the inferior parietal lobules. Functionally, it is considered to be a large-scale network of brain areas that form an integrated system for self-related cognitive activity, including autobiographical, self-monitoring, and social functions and for the mind-wandering process. ${ }^{12,16,31,37,40}$ Its rsFC has been shown to be decreased and disrupted after head injury, brain tumor, and various neuropsychiatric disorders such as schizophrenia, Alzheimer's disease, dementia disease, and epilepsy. ${ }^{8,27,39,40}$ Changes in this network after PNI have not been studied. We saw increased rsFC in the postcentral gyrus of the DMN in patients with BPI before surgery and a trend toward decreases in rsFC after surgery.

In our study, the postoperative patients who showed improvement experienced significant changes in rsFC compared with the group of postoperative patients with no improvement, which substantiates the notion that changes occur in the brain as a result of cortical plasticity and reorganization and is commensurate with the changes that occur in the peripheral nervous system along with its target innervation. Thus, there is a relationship between changes that occur in the periphery after a nerve injury and the changes seen in the cortex. Insufficient data exist to implicate one as the cause and the other as the effect; at best, the results of our study highlight only an association between the two. These changes are not limited to the motor and sensory cortical connections; such changes also occur at the higher cognitive levels. The DMN findings were in contrast to the $\mathrm{SN}$ changes. We found a reduction in DMN rsFC after surgery, although it was not statistically significant. It is a remote possibility that subtle anesthesia-related brain injury was a factor in the reduction in activity after 
surgery. We have no supporting evidence to implicate anesthesia for the changes, but it is well known that after anesthesia for cardiac and noncardiac extracranial surgeries, patients experience a period of cognitive decline (known as postoperative cognitive decline) that usually lasts for a few weeks up to 6 months or more. 25

Feng et al. ${ }^{10}$ studied changes in rs-fMRI results in patients with BPI who had not undergone surgery. They calculated a resting-state index, namely, amplitude of lowfrequency fluctuation. Along with changes in the sensorimotor cortex, they found activities in the regions related to higher cognition, especially in the right precuneus, calcarine/lingual gyri, and parahippocampal gyrus, that were lower than those in healthy controls.

We did not perform any neuropsychological tests before or after surgery to look for subtle cognitive changes in our patients. It is not known whether widespread clinically non-manifesting long-standing subtle changes in brain function and connections occur after surgery that manifest as changes in the RSNs and are yet to be identified. Further studies with rs-fMRI to associate neuropsychological results before and after surgery need to be performed.

We have shown that in patients with PNI, alterations in motor task-related and some unrelated RSNs and further changes in these networks were seen after surgery, depending on muscle power recovery. Before surgery, stress, pain, loss of motor cortex activity, and deafferentation of the sensory cortex might bring about cortical reorganization, resulting in changes in rs-fMRI signals. After surgery, if the patient's muscle power improves, cortical reorganization will happen again, and these changes were not seen in the postoperative patients who showed no improvement. What will be the natural course of these changes, whether they normalize, change, or remain the same, is not known and can be answered only through long-term follow up rsfMRI studies.

\section{Conclusions}

To our knowledge, ours is the first study to have found cortical plasticity in RSNs (i.e., SMN, SN, and DMN) in patients with BPI before and after successful or unsuccessful surgical repair. We found increased $\mathrm{rsFC}$ in the SMN and SN in patients whose muscle power improved after surgery, which indicates brain plasticity and network recovery after surgery. We also found increased rsFC in the DMN in patients with BPI, and that $\mathrm{rsFC}$ was reduced after surgery. In patients who did not improve after surgery, the RSNs were further reduced in activity after surgery. Further studies with neuropsychological assessments might be useful for increasing our understanding of the significance of these findings.

\section{Acknowledgments}

This research work was funded by the Department of Science and Technology, Government of India.

\section{References}

1. Anderson JS, Ferguson MA, Lopez-Larson M, YurgelunTodd D: Connectivity gradients between the default mode and attention control networks. Brain Connect 1:147-157, 2011
2. Beckmann CF, DeLuca M, Devlin JT, Smith SM: Investigations into resting-state connectivity using independent component analysis. Philos Trans R Soc Lond B Biol Sci 360:1001-1013, 2005

3. Beckmann CF, Mackay CE, Filippini N, Smith SM: Group comparison of resting-state FMRI data using multi-subject ICA and dual regression. Neuroimage 47 (Suppl 1):S148, 2009 (Poster)

4. Beckmann CF, Smith SM: Tensorial extensions of independent component analysis for multisubject FMRI analysis. Neuroimage 25:294-311, 2005

5. Cheng H, Shoung HM, Wu ZA, Chen KC, Lee LS: Functional connectivity of the transected brachial plexus after intercostal neurotization in monkeys. J Comp Neurol 380:155-163, 1997

6. Cohen LG, Bandinelli S, Findley TW, Hallett M: Motor reorganization after upper limb amputation in man. A study with focal magnetic stimulation. Brain 114 (Pt 1B):615-627, 1991

7. Damoiseaux JS, Rombouts SA, Barkhof F, Scheltens P, Stam CJ, Smith SM, et al: Consistent resting-state networks across healthy subjects. Proc Natl Acad Sci U S A 103:1384813853, 2006

8. Delnooz CC, Helmich RC, Toni I, van de Warrenburg BP: Reduced parietal connectivity with a premotor writing area in writer's cramp. Mov Disord 27:1425-1431, 2012

9. Ersland L, Rosén G, Lundervold A, Smievoll AI, Tillung T, Sundberg H, et al: Phantom limb imaginary fingertapping causes primary motor cortex activation: an fMRI study. Neuroreport 8:207-210, 1996

10. Feng JT, Liu HQ, Hua XY, Gu YD, Xu JG, Xu WD: Brain functional network abnormality extends beyond the sensorimotor network in brachial plexus injury patients. Brain Imaging Behav 10:1198-1205, 2016

11. Filippini N, MacIntosh BJ, Hough MG, Goodwin GM, Frisoni GB, Smith SM, et al: Distinct patterns of brain activity in young carriers of the APOE- $\varepsilon 4$ allele. Proc Natl Acad Sci U S A 106:7209-7214, 2009

12. Greicius MD, Krasnow B, Reiss AL, Menon V: Functional connectivity in the resting brain: a network analysis of the default mode hypothesis. Proc Natl Acad Sci U S A 100:253-258, 2003

13. Hsieh JC, Cheng H, Hsieh HM, Liao KK, Wu YT, Yeh TC, et al: Loss of interhemispheric inhibition on the ipsilateral primary sensorimotor cortex in patients with brachial plexus injury: fMRI study. Ann Neurol 51:381-385, 2002

14. Huang MC, Chen KC, Chuang TY, Chang WC, Lee LS, Huang WC, et al: Cervical root repair in adult rats after transection: recovery of forelimb motor function. Exp Neurol 180:101-109, 2003

15. Iwase Y, Mashiko T, Ochiai N, Kurosawa H: Postoperative changes on functional mapping of the motor cortex in patients with brachial plexus injury: comparative study of magnetoencephalography and functional magnetic resonance imaging. J Orthop Sci 6:397-402, 2001

16. Kim DH, Cho YJ, Tiel RL, Kline DG: Outcomes of surgery in 1019 brachial plexus lesions treated at Louisiana State University Health Sciences Center. J Neurosurg 98:1005-1016, 2003

17. Liu B, Li T, Tang WJ, Zhang JH, Sun HP, Xu WD, et al: Changes of inter-hemispheric functional connectivity between motor cortices after brachial plexuses injury: a restingstate fMRI study. Neuroscience 243:33-39, 2013

18. Lou L, Shou T, Li Z, Li W, Gu Y: Transhemispheric functional reorganization of the motor cortex induced by the peripheral contralateral nerve transfer to the injured arm. Neuroscience 138:1225-1231, 2006

19. Lundborg G: A 25-year perspective of peripheral nerve surgery: evolving neuroscientific concepts and clinical significance. J Hand Surg Am 25:391-414, 2000 
20. Malessy MJ, Bakker D, Dekker AJ, Van Duk JG, Thomeer RT: Functional magnetic resonance imaging and control over the biceps muscle after intercostal-musculocutaneous nerve transfer. J Neurosurg 98:261-268, 2003

21. Menon V, Uddin LQ: Saliency, switching, attention and control: a network model of insula function. Brain Struct Funct 214:655-667, 2010

22. Min Y, Park JW, Jin SU, Jang KE, Nam HU, Lee YS, et al: Alteration of resting-state brain sensorimotor connectivity following spinal cord injury: a resting-state functional magnetic resonance imaging study. J Neurotrauma 32:14221427, 2015

23. Moiyadi AV, Devi BI, Nair KP: Brachial plexus injuries: outcome following neurotization with intercostal nerve. J Neurosurg 107:308-313, 2007

24. Nagano A, Tsuyama N, Ochiai N, Hara T, Takahashi M: Direct nerve crossing with the intercostal nerve to treat avulsion injuries of the brachial plexus. J Hand Surg Am 14:980 985, 1989

25. Newman S, Stygall J, Hirani S, Shaefi S, Maze M: Postoperative cognitive dysfunction after noncardiac surgery: a systematic review. Anesthesiology 106:572-590, 2007

26. Nichols TE, Holmes AP: Nonparametric permutation tests for functional neuroimaging: a primer with examples. Hum Brain Mapp 15:1-25, 2002

27. Norton L, Hutchison RM, Young GB, Lee DH, Sharpe MD, Mirsattari SM: Disruptions of functional connectivity in the default mode network of comatose patients. Neurology 78:175-181, 2012

28. Pawela CP, Biswal BB, Hudetz AG, Li R, Jones SR, Cho YR, et al: Interhemispheric neuroplasticity following limb deafferentation detected by resting-state functional connectivity magnetic resonance imaging (fcMRI) and functional magnetic resonance imaging (fMRI). Neuroimage 49:2467-2478, 2010

29. Qiu TM, Chen L, Mao Y, Wu JS, Tang WJ, Hu SN, et al: Sensorimotor cortical changes assessed with resting-state fMRI following total brachial plexus root avulsion. J Neurol Neurosurg Psychiatry 85:99-105, 2014

30. Reddy H, Bendahan D, Lee MA, Johansen-Berg H, Donaghy $\mathrm{M}$, Hilton-Jones D, et al: An expanded cortical representation for hand movement after peripheral motor denervation. J Neurol Neurosurg Psychiatry 72:203-210, 2002

31. Ros T, Théberge J, Frewen PA, Kluetsch R, Densmore M, Calhoun VD, et al: Mind over chatter: plastic up-regulation of the fMRI salience network directly after EEG neurofeedback. Neuroimage 65:324-335, 2013

32. Rosazza C, Minati L: Resting-state brain networks: literature review and clinical applications. Neurol Sci 32:773-785, 2011

33. Smith SM: Fast robust automated brain extraction. Hum Brain Mapp 17:143-155, 2002
34. Smith SM, Jenkinson M, Woolrich MW, Beckmann CF, Behrens TE, Johansen-Berg $\mathrm{H}$, et al: Advances in functional and structural MR image analysis and implementation as FSL. Neuroimage 23 (Suppl 1):S208-S219, 2004

35. Smith SM, Nichols TE: Threshold-free cluster enhancement: addressing problems of smoothing, threshold dependence and localisation in cluster inference. Neuroimage 44:83-98, 2009

36. Sokki AM, Bhat DI, Devi BI: Cortical reorganization following neurotization: a diffusion tensor imaging and functional magnetic resonance imaging study. Neurosurgery 70:13051311,2012

37. Spreng RN, Grady CL: Patterns of brain activity supporting autobiographical memory, prospection, and theory of mind, and their relationship to the default mode network. J Cogn Neurosci 22:1112-1123, 2010

38. van den Heuvel MP, Hulshoff Pol HE: Exploring the brain network: a review on resting-state fMRI functional connectivity. Eur Neuropsychopharmacol 20:519-534, 2010

39. van Eimeren T, Monchi O, Ballanger B, Strafella AP: Dysfunction of the default mode network in Parkinson disease: a functional magnetic resonance imaging study. Arch Neurol 66:877-883, 2009

40. Vanhaudenhuyse A, Noirhomme Q, Tshibanda LJF, Bruno MA, Boveroux P, Schnakers C, et al: Default network connectivity reflects the level of consciousness in non-communicative brain-damaged patients. Brain 133:161-171, 2010

\section{Disclosures}

The authors report no conflict of interest concerning the materials or methods used in this study or the findings specified in this paper.

\section{Author Contributions}

Conception and design: Devi. Acquisition of data: Bhat, Bharti, Panda. Analysis and interpretation of data: Bharti, Panda. Drafting the article: Bhat. Critically revising the article: Bhat, Devi.

\section{Supplemental Information}

\section{Online-Only Content}

Supplemental material is available online.

Supplemental Table 1. https://thejns.org/doi/suppl/10.3171/ 2016.12.FOCUS16430.

\section{Correspondence}

Dhananjaya I. Bhat, Department of Neurosurgery, National Institute of Mental Health and Neurosciences, Bangalore 560029, India.email: dibhat@rediffmail.com. 